\title{
ЭВОЛЮЦИЯ ЭНЕРГЕТИКИ С ЗАМЕЩЕНИЕМ ТРАДИЦИОННЫХ ИСТОЧНИКОВ ЭНЕРГИИ, РАССЧИТАННАЯ МЕТОДОМ НЕЧЕТКОЙ ЛОГИКИ
}

\author{
(C) 2021 Вознесенская Дарья Дмитриевна \\ Высшая инженерно-экономическая школа \\ Санкт-Петербургский политехнический университет Петра Великого, Россия, Санкт-Петербург \\ E-mail:vdaria0722@gmail.com \\ (c) 2021 Лопырев Илья Андреевич \\ Высшая инженерно-экономическая школа \\ Санкт-Петербургский политехнический университет Петра Великого, Россия, Санкт-Петербург \\ E-mail: ilyalo1808@mail.ru
}

\section{(c) 2021 Новикова Ольга Валентиновна}

Высшая инженерно-экономическая школа, кандидат экономических наук

Санкт-Петербургский политехнический университет Петра Великого, Россия, Санкт-Петербург

E-mail: novikova-olga1970@yandex.ru

\section{(C) 2021 Конников Евгений Александрович}

Высшая инженерно-экономическая школа, кандидат экономических наук

Санкт-Петербургский политехнический университет Петра Великого, Россия, Санкт-Петербург

E-mail: konnikov.evgeniy@gmail.com

В данной статье рассматривается проблема отсутствия эмпирической составляющей в прогнозировании развития энергетической отрасли России с учетом известных трендов эволюции. Авторы, с помощью метода нечеткой логики, рассчитали показатель «Степень замещения» традиционных источников энергии АЭС, ТЭС, ГЭС на нетрадиционные возобновляемые источники (НВИЭ) с 2000 по 2020 год в России, в сравнении с Германией, Японией, Китаем и США. Данный показатель может быть применён при прогнозировании развития ТЭК для получения более точных результатов.

Ключевые слова. нечёткая логика, традиционная энергетика, нетрадиционные возобновляемые источники энергии, НВИЭ, степень замещения, прогноз развития ТЭК, эволюция энергетики, АЭС, ТЭС, ГЭС.

Введение. Тема эволюции энергетики в последние годы объективно рассматривается с учетом активного роста доли возобновляемых источников энергии. И важны не только фактически реализованные проекты, но и активность проявляемого интереса к новым технологиям. Экологизация новой энергетической политики всех стран чаще определяется нетрадиционными ВИЭ, к которым относятся ветровые, солнечные, геотермальные, приливные, био-, волновые, малые гидро- электрические станции, а также энергия от сжигания мусора и утилизации свалочного газа и др. [1] Особенности расположения территорий с высоким потенциалом конкретных ресурсов определяют и целесообразность создания новых проектов. Не любая территория с учетом уровня и структуры спроса на электрическую и тепловую энергию потенциально сможет в ближайшие 10-20 лет отказаться от традиционных углеводородов. В контексте данной тенденции, особенно актуальным является прогнозирование степени замещения традиционных источников энергии НВИЭ. Однако, исследований по данной теме, которые рассматривают замещение мощностей с учетом региональной специфики в динамике с 2000 по 2020 годы, практически отсутствуют.

В процессе исследования был произведен обзор научных работ по теме. Акцент при изучении существующих материалов был сделан на статьи, где присутствуют элементы моделирования и/или анализа статистической информации для определения динамики и прогнозирования развития генерации на основе НВИЭ, предпочтительно, с учетом региональной специфики.

Так, в статье [2] были проанализированы факторы, влияющие на развитие возобновляемых источников энергии в нескольких странах: Бразилии, Индии, Китае, России и ЮАР. Для целей исследования были использованы методы FEVD и 
PCSE, с временным рядом данных с 1990 по 2010 год. Показатели условно разделены на 3 категории: политические, социально-экономические и специфические для каждого государства.

В работе [3] анализируются движущие силы, способствующие использованию возобновляемых источников энергии на основе водорода. Оценка производится для 32 стран с использованием панельных данных. Реляционный анализ Грея используется для изучения взаимосвязей на национальном уровне между развитием возобновляемой энергетики и факторами, на которые она влияет.

В исследовании [4] приводится прогноз развития генерации на основе возобновляемых источников в Китае. Также оценено влияние ВИЭ на изменение политики контроля выбросов CO2. Таким образом, в работе устанавливается тесная связь между развитием возобновляемых источников энергии, снижением выбросов диоксида углерода и изменением законодательства. Особенно явно эта взаимосвязь проявляется на фоне того, что КНР - одна из главных загрязнителей планеты, притом, ввиду ускоренных темпов роста промышленности и других факторов, до недавнего времени не особенно заинтересованная в снижении негативного влияния на окружающую среду.

В статьях [5] и [6] рассматривается инвестиционная привлекательность различных технологий возобновляемых источников энергии в КНР. В данных исследованиях используется альтернативный стандартной методике способ оценки - опционный, который, по утверждениям авторов статьи, позволяет более точно оценить проекты, с учетом вероятностного подхода и гибкости менеджмента.

Работа [7] предлагает к рассмотрению стохастическую модель, которая отражает различные возможности оптимизации структуры генерирующих мощностей с учетом внедрения НВИЭ и эффектов от таких изменений. Основное преимущество этой модели состоит в том, что она эффективно расширяет пространство выбора для лиц, принимающих решения, и обеспечивает более сбалансированный план оптимизации. Было выявлено множество схем освоения возобновляемых источников энергии под взаимным влиянием различных комбинаций весов и уровней удовлетворенности ограничениями. Расчет выработки электроэнергии с учетом изменения метеорологических факторов был включен в предложенную модель оптимизации, которая эффективно предотвращает потенциальный дисбаланс между спросом и предложением электроэнергии в условиях изменения климата. Расчет произведен для города Далиан в Китае, однако, возможно, модифицированная версия модели может быть применена в других регионах.

Научная работа [8] иллюстрирует различные дополнительные подходы к традиционной модели планирования электроэнергии, чтобы учесть различные характеристики возобновляемых источников энергии, и использует энергетический сектор Тайваня в качестве примера. Теория портфеля, теория кривой обучения и кредит мощности применяются в предлагаемой модели для отражения характеристик возобновляемых источников энергии, таких как защита от волатильности цен на ископаемое топливо, значительный технический прогресс и периодическая генерация. Результаты моделирования демонстрируют, что использование возобновляемых источников энергии имеет преимущество, заключающееся в защите от рисков неустойчивых цен на ископаемое топливо, а также в сокращении выбросов диоксида углерода.

Результаты исследования достаточно обширно описывают влияние факторов внешней среды на динамику инвестиций в ВИЭ и изменения в регулирующем его законодательстве. Однако, в моделях, использованных для анализа, не учтены возможные эффекты от замещения традиционных источников с помощью ВИЭ, так как они изначально противопоставляются друг другу.

Таким образом, рассчитанные в данной работе данные могут стать базой для прогнозирования развития энергетики России с учетом развития генерации с использованием нетрадиционных возобновляемых источников энергии.

Целью исследования является предложить дополнить показателем «степень замещения» при оценке зависимости между развитием НВИЭ и динамикой показателей традиционных источников генерации энергии для получения более точных прогнозных значений баланса выработки электрической энергии.

Выбор метода нечеткой логики обусловлен тем, что используемые факторы могут быть как количественными, так и качественными. Количественные оценки основываются на статистической информации, хотя часто приходится сталкиваться с проблемой неполноты и недостаточности такого типа информации. Качественные оценки риска основаны, зачастую на экспертных оценках. А для возможности ка- 
чественного анализа количественных и качественных показателей может быть использован метод построения нечетких моделей.

Для анализа степени замещения традиционных источников электрической и тепловой энергии, к которым относятся АЭС, ТЭС и даже крупные ГЭС, нетрадиционными возобновляемыми источниками (НВИЭ) методом нечетких множеств были взяты 3 количественных фактора, отображающих особенности энергетики пяти стран: России, Китая, Японии, Германии и США. Степень замещения - это интегральный показатель, отражающий сложность и целесообразность замещения традиционных генерирующих мощностей данного типа НВИЭ по шкале от 0 до 1. Выбранные факторы [9]:

1. $\mathrm{V}_{\mathrm{CO}_{2}}$ - выбросы $\mathrm{CO}_{2}$ от производства электро- и теплоэнергии, всего (\% от общего количества выбросов промышленностью) - для всех групп традиционных источников энергии;

2. W - производство электроэнергии (в \% от общей выработки по каждому типу электростанций);

3. N - установленные мощности по каждому типу электрических станций (в МВт).

Bсе показатели были взяты в динамике с 2000 по 2020 годы. Данные факторы выбраны, так как отражают наиболее вероятные направления влияния замещения традиционных генерирующих мощностей с помощью НВИЭ. Так, в краткосрочной перспективе их влияние выражается, в большей степени, в снижении выработ- ки традиционными электростанций [10]. Далее, снижается объем выбросов $\mathrm{CO}_{2}$ от стационарных энергетических источников, так как мощности НВИЭ практически не выделяют диоксид углерода [10]. И, в среднесрочной перспективе, изменения структуры генерации может сопровождаться тем, что выводимое из эксплуатации оборудование заменяется НВИЭ, а не более новыми образцами аналогичного типа. Примером может служить энергетическая политика Германии в отношении АЭС [11].

Каждый из факторов имеет свои, существенно отличающиеся от других единицы измерения [12]. Соответственно, для получения несмещенных оценок требуется нормализовать данные с приведением к шкале от 0 до 1 для возможности совместной оценки факторов и учета характера их влияния на проект. Для нормализации собранной по России статистики, в качестве дополнительных значений, использованы аналогичные данные по четырем другим странам за тот же период. Все значения нормализованы относительно среднего арифметического по соответствующему показателю. В качестве примера проведения расчета представлены данные за 2020 г. Так, в таблице 1 приведен исходный вид расчетных показателей для ГЭС.

Далее каждый фактор располагается на шкале от 0 до 1 в зависимости от того, в какие отрезки шкалы попадает его значение. Притом, для каждого отрезка рассчитывается своя «вероятность» нахождения фактора (таблица 2).

Таблица 1. Исходный вид расчётных показателей для ГЭС

\begin{tabular}{|l|c|c|c|}
\hline \multicolumn{1}{|c|}{2020} & $\begin{array}{l}\mathrm{V}_{\mathrm{CO} 2}, \text { \% от общего } \\
\text { сжигания топлива }\end{array}$ & $\mathrm{W}$, \% от общего объема & $\mathrm{N}, \mathrm{MBT}$ \\
\hline Китай & 57,5 & 20,1 & 366871,3 \\
\hline Германия & 43,8 & 3 & 11723,5 \\
\hline Япония & 63,2 & 7,7 & 51401,3 \\
\hline Россия & 54 & 14,8 & 52611,6 \\
\hline США & 39,8 & 5,4 & 105485,2 \\
\hline $\begin{array}{l}\text { Нормированное значение } \\
\text { показателя по РФ }\end{array}$ & 0,606838 & 0,690058 & 0,11513 \\
\hline
\end{tabular}

Таблица 2. Распределение значений факторов по шкале нечетких множеств для ГЭС

\begin{tabular}{|c|c|c|c|c|c|c|}
\hline 2020 & $0-0,333$ & $0,167-0,5$ & $0,333-0,67$ & $0,5-0,833$ & $0,67-1$ & $\begin{array}{c}\text { Расчетное } \\
\text { 3начение } \\
\text { показателя }\end{array}$ \\
\hline $\mathrm{V}_{\mathrm{CO} 2}$ & & & & 0,679166 & 0,320834 & 0,606838 \\
\hline $\mathrm{W}$ & & & 0,305615 & 0,694385 & & 0,39823 \\
\hline $\mathrm{N}$ & 0,749527 & 0,250473 & & & & 0,080408 \\
\hline
\end{tabular}


После этого каждому фактору присваивается весовой коэффициент. В данном случае, как принято стандартной методикой оценки с использованием нечетких множеств, весовые значения рассчитаны с использованием экспертных оценок [13, 14].

Для каждой отрасли были определены свои оценки весов, чтобы отобразить в итоговых значениях различную значимость каждого из использованных в расчетах факторов. К примеру, объемы выбросов $\mathrm{CO}_{2}$ имеют критичную важность для оценки возможности замещения ТЭС нетрадиционными возобновляемыми источниками энергии. С другой стороны, для оценки показателя замещения ГЭС этот фактор не имеет такого значения, ввиду большей экологичности гидроэлектростанций. Таким образом, весовые коэффициенты в данном исследовании выполняют не только роль балансировки факторов внутри одной оцениваемой отрасли, но и позволяют модифицировать расчеты для более точного отражения влияния факторов на каждый тип генерирующих мощностей, к которому они применены $[15,16]$.

Так, для анализа ГЭС наибольший коэффициент $(0,5)$ присвоен показателю «Производство электроэнергии», так как он является наиболее репрезентативным и наглядно показывает смещение энергетики с выработки энергии традиционными источниками на НВИЭ, далее пока- зателю «Установленная мощность» присвоен коэффициент 0,3, а фактору «Выбросы $\mathrm{CO}_{2}$ » $-0,1$, так как у данного типа ЭС выбросы углекислого газа практически вовсе отсутствуют [17]. Данные представлены в таблице 3.

Аналогично проводятся расчеты для ТЭС и АЭС. Стоит отметить, что весовые коэффициенты для расчета показателя для ТЭС расставлялись исходя из того, что на замещение данного типа станций НВИЭ в большей степени влияет показатель «Выбросы $\mathrm{CO}_{2}$ », так как это главный недостаток ТЭС, соответственно присвоенный коэффициент составляет 0,45. Далее, как и в случае с ГЭС, наиболее репрезентативным является фактор «Производство электроэнергии», который имеет вес 0,35 , и последним по степени оказанного влияния является «Установленная мощность» с весом 0,2 [18]. Таким образом, в таблицах 4-6 представлен расчет степени замещения ТЭС НВИЭ за 2020 год.

Весовые коэффициенты для расчета показателя для АЭС принимались исходя из того, что выбросы $\mathrm{CO}_{2}$ от данного типа станций больше, чем от ГЭС, но меньше, чем от ТЭС, соответственно данный показатель имеет вес 0,3. Производство электрической энергии в\% от общей выработки имеет наибольший коэффициент, так как наиболее эффективно отражает тенденцию замещения АЭС НВИЭ и имеет вес 0,5. В свою очередь, коэффициент показателя «Установлен-

Таблица 3. Расчёт итогового значения показателя «Степень замещения» с использованием узловых точек для ГЭС

\begin{tabular}{|c|c|c|c|c|c|c|}
\hline 2020 & $0-0,333$ & $0,167-0,5$ & $0,333-0,67$ & $0,5-0,833$ & $0,67-1$ & Bес \\
\hline $\mathrm{V}_{\mathrm{CO} 2}$ & 0 & 0 & 0 & 0,067917 & 0,032083 & 0,10 \\
\hline $\mathrm{W}$ & 0 & 0 & 0,18336921 & 0,183369 & 0,41663 & 0,6 \\
\hline $\mathrm{N}$ & 0,22486 & 0,075142 & 0 & 0 & 0 & 0,3 \\
\hline $\begin{array}{c}\text { Узловая } \\
\text { оценка }\end{array}$ & 0,18731 & 0,05012 & 0,091684605 & 0,083678 & 0,07494 & $\mathbf{0 , 4 8 7 7 2}$ \\
\hline
\end{tabular}

Таблица 4. Исходный вид расчётных показателей для ТЭС

\begin{tabular}{|l|c|c|c|}
\hline \multicolumn{1}{|c|}{2020} & $\begin{array}{l}\mathrm{V}_{\mathrm{CO} 2} \text {, \% от общего } \\
\text { сжигания топлива }\end{array}$ & W, \% от общего объема & $\mathrm{N}, \mathrm{MBT}$ \\
\hline Китай & 57,5 & 67,8 & 1214472 \\
\hline Германия & 43,8 & 51,1 & 105340,8 \\
\hline Япония & 63,2 & 85 & 194523,3 \\
\hline Россия & 54 & 64,6 & 746749,6 \\
\hline США & 39,8 & 62,6 & 0,080408 \\
\hline $\begin{array}{l}\text { Нормированное значение } \\
\text { показателя по РФ }\end{array}$ & 0,606838 & 0,39823 \\
\hline
\end{tabular}


Таблица 5. Распределение значений факторов по шкале нечетких множеств для ТЭС

\begin{tabular}{|c|c|c|c|c|c|c|}
\hline 2020 & $0-0,333$ & $0,167-0,5$ & $0,333-0,67$ & $0,5-0,833$ & $0,67-1$ & $\begin{array}{c}\text { Расчетное } \\
\text { значение } \\
\text { показателя }\end{array}$ \\
\hline $\mathrm{V}_{\mathrm{CO} 2}$ & & & 0,18967686 & 0,8103231 & & 0,606838 \\
\hline $\mathrm{W}$ & & 0,30561535 & 0,69438465 & & & 0,39823 \\
\hline $\mathrm{N}$ & 0,7585358 & 0,24146416 & & & & 0,080408 \\
\hline
\end{tabular}

Таблица 6. Расчёт итогового значения показателя «Степень замещения» с использованием узловых точек для ГЭС

\begin{tabular}{|c|c|c|c|c|c|c|}
\hline 2020 & $0-0,333$ & $0,167-0,5$ & $0,333-0,67$ & $0,5-0,833$ & $0,67-1$ & Вес \\
\hline $\mathrm{V}_{\mathrm{CO} 2}$ & 0 & 0 & 0,0853546 & 0,36465 & 0 & 0,45 \\
\hline $\mathrm{W}$ & 0 & 0,106965372 & 0,2430346 & 0 & 0 & 0,35 \\
\hline $\mathrm{N}$ & 0,15171 & 0,048292832 & 0 & 0 & 0 & 0,2 \\
\hline $\begin{array}{c}\text { Узловая } \\
\text { оценка }\end{array}$ & 0,12637 & 0,103557223 & 0,1641946 & 0,12143 & 0 & $\mathbf{0 , 5 1 5 5 5}$ \\
\hline
\end{tabular}

ная мощность» равен 0,2 . Таким образом, в таблицах 7-9 представлен расчет степени замещения АЭС НВИЭ за 2020 год.

Следующий этап - определение узловых точек шкалы. Если факторы влияют на проект позитивно, то значение каждой последующей узловой точки увеличивается. Если негативно уменьшается.

После этого вероятностные значения умножаются на соответствующие их факторам весовые коэффициенты и располагаются на шкале от 0 до 1 в соответствии с расположением на предыдущей шкале. Далее, полученные значения суммируются по отрезкам на шкале и умножаются на коэффициенты узловых точек. Полученные таким образом произведения складываются, образовывая итоговую оценку риска, которая интерпретируется в зависимости от определенного ранее изменения узловых точек на шкале (таблицы 3, 6, 9). Также в таблице 10 представлены итоговые значения степени замещения ЭС с помощью НВИЭ за 2000-2020 гг.

Для формирования итоговой шкалы нечет- ких значений переменной Ү следует сначала ранжировать факторы по значимости их влияния на результирующий показатель. В данной работе ранжирование проводилось в соответствии с весовыми коэффициентами, присвоенными ранее.

В таблице 11 представлена итоговая шкала нечетких значений переменной Y, позволяющая преобразовать количественную оценку рисков, получившуюся в результате применения метода нечетких множеств, в конкретное описание, в соответствие с тем, в какие множества значений попадает итоговая оценка.

Что касается динамики показателей, представленных в таблице 10, каждая из исследуемых отраслей имеет собственные особенности изменения показателя замещения.

Показатели теплоэлектростанций стабильно входят в промежутки $[0,167-0,5]$ и $[0,333-0,67]$ итоговой шкалы. В большей степени (от $75 \%$ до 96\%) итоговые значения принадлежат к отрезку $[0,333-0,67]$, что позволяет утверждать, что степень замещения генерирующих мощностей

Таблица 7. Исходный вид расчётных показателей для АЭС

\begin{tabular}{|l|c|c|c|}
\hline \multicolumn{1}{|c|}{2020} & $\begin{array}{l}\mathrm{V}_{\mathrm{CO} 2} \text {, \% от общего } \\
\text { сжигания топлива }\end{array}$ & W, \% от общего объема & N, MBт \\
\hline Китай & 57,5 & 2,9 & 45076 \\
\hline Германия & 43,8 & 16 & 10341,6 \\
\hline Япония & 63,2 & 0 & 36831,5 \\
\hline Россия & 54 & 18,8 & 29799,5 \\
\hline США & 39,8 & 20,8 & 102095,3 \\
\hline $\begin{array}{l}\text { Нормированное значение } \\
\text { показателя по РФ }\end{array}$ & 0,606838 & 0,903846 & 0,212067 \\
\hline
\end{tabular}


Таблица 8. Распределение значений факторов по шкале нечетких множеств для АЭС

\begin{tabular}{|c|c|c|c|c|c|c|}
\hline 2020 & $0-0,333$ & $0,167-0,5$ & $0,333-0,67$ & $0,5-0,833$ & $0,67-1$ & $\begin{array}{c}\text { Расчетное } \\
\text { значение } \\
\text { показателя }\end{array}$ \\
\hline $\mathrm{V}_{\mathrm{CO} 2}$ & & & & 0,679166 & 0,320834 & 0,606838 \\
\hline $\mathrm{W}$ & & & & & 1 & 0,903846 \\
\hline $\mathrm{N}$ & 0,363163 & 0,636837 & & & & 0,212067 \\
\hline
\end{tabular}

Таблица 9. Расчёт итогового значения показателя «Степень замещения» с использованием узловых точек для АЭС

\begin{tabular}{|c|c|c|c|c|c|c|}
\hline 2020 & $0-0,333$ & $0,167-0,5$ & $0,333-0,67$ & $0,5-0,833$ & $0,67-1$ & Bec \\
\hline $\mathrm{V}_{\mathrm{CO} 2}$ & 0 & 0 & 0 & 0,20375 & 0,09625 & 0,30 \\
\hline $\mathrm{W}$ & 0 & 0 & 0 & 0 & 0,5 & 0,5 \\
\hline $\mathrm{N}$ & 0,0726 & 0,1274 & 0 & 0 & 0 & 0,2 \\
\hline $\begin{array}{c}\text { Узловая } \\
\text { оценка }\end{array}$ & 0,0605 & 0,0850 & 0 & 0,067849 & 0,099574 & $\mathbf{0 , 3 1 2 8 8}$ \\
\hline
\end{tabular}

Таблица 10. Степень замещения традиционных ЭС с помощью НВИЭ, рассчитанная по методу нечеткой логики

\begin{tabular}{|c|c|c|c|}
\hline Год & ТЭС & ГЭС & АЭС \\
\hline 2000 & 0,463699329 & 0,46369933 & 0,4508143 \\
\hline 2001 & 0,474571857 & 0,28247544 & 0,446498 \\
\hline 2002 & 0,486856388 & 0,28254927 & 0,3972165 \\
\hline 2003 & 0,50520823 & 0,28199318 & 0,3924711 \\
\hline 2004 & 0,495765296 & 0,28630798 & 0,4007771 \\
\hline 2005 & 0,485731504 & 0,31725403 & 0,3968885 \\
\hline 2006 & 0,478776518 & 0,32283967 & 0,3948264 \\
\hline 2007 & 0,491189893 & 0,3281405 & 0,3691568 \\
\hline 2008 & 0,457588129 & 0,33425779 & 0,3746021 \\
\hline 2009 & 0,474167208 & 0,33793537 & 0,3839976 \\
\hline 2010 & 0,457468799 & 0,34067849 & 0,3793154 \\
\hline 2011 & 0,465491924 & 0,34267998 & 0,2870375 \\
\hline 2012 & 0,461488569 & 0,3431001 & 0,2855133 \\
\hline 2013 & 0,470303527 & 0,34540688 & 0,2850618 \\
\hline 2014 & 0,433034814 & 0,34678621 & 0,2849444 \\
\hline 2015 & 0,423870992 & 0,35899988 & 0,2826082 \\
\hline 2016 & 0,428779602 & 0,3664451 & 0,2817912 \\
\hline 2017 & 0,42220122 & 0,37342772 & 0,2809477 \\
\hline 2018 & 0,445656563 & 0,38394436 & 0,2915965 \\
\hline 2019 & 0,458070945 & 0,39395949 & 0,3032508 \\
\hline 2020 & 0,515550823 & 0,487724 & 0,31288 \\
\hline
\end{tabular}


Таблица 11. Шкала нечетких значений переменной Y

\begin{tabular}{|c|c|c|}
\hline $\begin{array}{c}\text { Множество } \\
\text { значений }\end{array}$ & Наименование & Описание \\
\hline $0-0,333$ & Высокое замещение & $\begin{array}{l}\text { Постоянно снижающийся объем выработки, используемой установ- } \\
\text { ленной мощности и выбросов углекислого газа свидетельствует о } \\
\text { снижении использования данного вида генерирующих мощностей в } \\
\text { России. Приоритет важности остается за показателями объема выбро- } \\
\text { сов и выработки энергии, так как снижение установленной мощно- } \\
\text { сти происходит с временной задержкой к изменениям в структуре } \\
\text { отраслей. }\end{array}$ \\
\hline $0,167-0,5$ & $\begin{array}{c}\text { Замещение выше } \\
\text { среднего }\end{array}$ & $\begin{array}{l}\text { Ввиду планомерного снижения выработки и выбросов СО2 в атмос- } \\
\text { феру можно сделать вывод, что структура генерирующих мощностей } \\
\text { изменяется в сторону наращивания доли НВИЭ с темпами, превыша- } \\
\text { ющими средние среди развитых стран. }\end{array}$ \\
\hline $0,333-0,667$ & Замещение среднее & $\begin{array}{l}\text { Изменения основных показателей, использованных в анализе, свиде- } \\
\text { тельствуют о постепенном увеличении доли НВИЭ в структуре выра- } \\
\text { ботки. Однако, эти изменения не носят массового характера, так как } \\
\text { присутствуют факторы, сдерживающие процесс перехода к зеленой } \\
\text { энергетике. }\end{array}$ \\
\hline $0,5-0,833$ & $\begin{array}{c}\text { Замещение ниже } \\
\text { среднего }\end{array}$ & $\begin{array}{l}\text { Изменения в структуре генерации имеют исключительно локальных } \\
\text { характер, так как текущее социально-экономическое положение, тех- } \\
\text { нологические и инфраструктурные ограничения не позволяют стране } \\
\text { более масштабно реформировать отрасль для развития генерации на } \\
\text { основе НВИЭ. }\end{array}$ \\
\hline $0,667-1$ & Замещение низкое & $\begin{array}{l}\text { Высокий уровень выбросов, особенно влияющий при оценке ТЭС, } \\
\text { делает генерирующие мощности отрасли приоритетеным кандидатом } \\
\text { на замещение с помощью различных НВИЭ. Однако большая сово- } \\
\text { купная установленная мощность и существенная доля данного вида } \\
\text { электростанций в структуре генерации делает невозможным полно- } \\
\text { масштабный вереход на традиционные источники при нынешнем } \\
\text { уровне развития технологий. }\end{array}$ \\
\hline
\end{tabular}

ТЭС в России находится на среднем уровне по сравнению с странами развитого мира. Среди всех исследованных отраслей, ТЭС имеют самые негативные оценки замещения по нескольким причинам: ТЭС занимают около 64\% в энергобалансе РФ, поэтому, резкий отказ от данного типа ЭС в нашей стране невозможен. Также следует отметить, что энергия, вырабатываемая ТЭС в той или иной степени участвует в покрытии всех зон графика нагрузки энергосистемы. Еще одни преимуществом ТЭС над НВИЭ является возможность производства тепловой энергии, а также низкие требования к условиям географического расположения станций. Недостаток ТЭС в контексте вредных выбросов $\mathrm{CO}_{2}$, и как следствие возможности замещения их НВИЭ, компенсируется тем, что большая их часть, 70\%, уже сейчас переведена с твердого и дизельного топлива на природный газ, который, согласно оценкам ведущих специалистов компании ПАО «Газпром» [19], отвечает всем требованиям по снижению вредных выбросов в атмосферу. Также стоит отметить, что Россия, будучи одним из главных производителей и экспортеров ископаемых топлив, в ближайшее время не планирует отказа от их добычи и использования.

Показатели замещения ГЭС показывают более высокие результаты: степень замещения входит в промежутки [0-0,333] и [0,167-0,5] («высокое замещение» и «замещение выше среднего») с 2000 года по 2008, и только после 2009 года показатели перемещаются к отрезкам [0,167-0,5] и [0,333-0,67] («замещение выше среднего» и «среднее замещение»). Это значит, что с развитием энергетики России становится все сложнее отказаться от выработки энергии с помощью ГЭС. Некоторые из причин этого следующие: крайне дешевая электроэнергия, практически полное отсутствие выбросов в атмосферу, использование воды в качестве «топлива» для получения энергии. В дополнение в перечисленному выше, высокая маневренность и установленная мощность современных ГЭС позволяет использовать их для покрытия и базовой и пиковой части графика нагрузки. Однако, недостатки гидроэлектростанций, такие как: невозможность производства тепловой энергии, сложность полного останова, зависимость от осадков и сезонной водности и серьезное негативное влияние на экосистемы вокруг заставляют задуматься о 
применении альтернативных источников энергии.

АЭС имеют наиболее высокие оценки по показателю замещения. С 2011 года итоговое значение попадает в отрезки [0-0,333] и [0,167-0,5], что позволяет утверждать, что атомные электростанции наиболее пригодны для замещение с помощью НВИЭ по нескольким причинам: ограничения по расположению исключительно вдали от населенных пунктов, низкая маневренность, крайне высокие требования к безопасности при эксплуатации, большое количество крайне радиоактивных отходов и сниженный после нескольких ключевых катастроф за последние 50 лет социальный имидж отрасли. Все эти недостатки приводят к тому, что некоторые развитые страны, в частности, Япония и Германия, активно отказываются от АЭС в своей структуре генерирующих мощностей. В случае России постепенный отказ от АЭС также возможен ввиду относительно небольшой их доли в структуре генерации страны.

Заключение. В данной работе была проведена оценка степени замещения традиционных источников энергии с помощью НВИЭ за 20 лет в нескольких странах.

Основным итогом анализа методом нечеткой логики являются интегральные показатели для трех основных отраслей традиционной генерации, отражающие структурные изменения в энергетике России при увеличении мощностей НВИЭ по сравнению с США, Германией, Япони- ей и Китаем - странами лидерами по темпам внедрения НВИЭ. Теплоэнергетика получила наихудшие оценки из всех оцененных отраслей, несмотря на ее неэкологичность, так как является основой ЕЭС России, что усложняет ее замену нетрадиционными источниками энергии. Однако, подобные реформы необходимы в определенном масштабе, чтобы снизить объем выбросов $\mathrm{CO}_{2}$ в атмосферу.

Что касается гидроэнергетики, ее средние оценки проистекают из важности ГЭС в региональных энергосистемах Российской Федерации. При этом гидроэлектростанции являются ВИЭ, а значит, несмотря на некоторые их недостатки, в том числе экологические, не требуют масштабной замены.

Атомная энергетика получила наилучшие оценки, что говорит о возможности ее безболезненного замещения более экологичными источниками энергии. Тем не менее, с главной проблемой АЭС - утилизацией отходов после вывода станций из эксплуатации, России только предстоит встретиться.

Результаты данной работы можно использовать как базу дальнейших исследований в области нетрадиционных источников энергии, в частности, для выявления частных тенденций в описанных странах, которые влияют на динамику оценок. Также, интегральные показатели, полученные методом нечетких множеств, можно применить для построения прогноза развития энергетики Российской Федерации.

\section{Библиографический список}

1. Прогноз развития энергетики мира и России 2019 / под ред. А.А. Макарова, Т. А. Митровой, В.А. Кулагина; ИНЭИ РАН-Московская школа управления СКОЛКОВО - Москва, 2019.- 210 с. - ISBN 978-5-91438-028-8

2. Aguirre M. / Determinants of renewable energy growth: A global sample analysis / Mariana Aguirre, Gbenga Ibikunle / Energy Policy, Volume 69, 2014 - C. 374-384.

3. Wang B. / How does hydrogen-based renewable energy change with economic development? Empirical evidence from 32 countries / Bing Wang, Zhifu Mi, Ioan Nistor, Xiao-Chen Yuan / International Journal of Hydrogen Energy / Volume 43, Issue 25, 2018 - C. 11629-11638.

4. Liu T. / Development forecast of renewable energy power generation in China and its influence on the GHG control strategy of the country / Tong Liu, Gang Xu, Peng Cai, Longhu Tian, Qili Huang / Renewable Energy, Volume 36, Issue 4, 2011.- C. 1284-1292.

5. Santos L. / Real Options versus Traditional Methods to assess Renewable Energy Projects / Lúcia Santos, Isabel Soares, Carla Mendes, Paula Ferreira / Renewable Energy, Volume 68, 2014. - C. 588-594.

6. Zhang M. M. / A real options model for renewable energy investment with application to solar photovoltaic power generation in China / M. M.Zhang, P. Zhou, D. Q.Zhou / Energy Economics, Volume 59, 2016. - C. $213-226$.

7. Meng $\mathrm{Na} / \mathrm{A}$ stochastic multi-objective optimization model for renewable energy structure adjustment management - A case study for the city of Dalian, China / Na Meng, Ye Xu, GuoHe Huang / Ecological Indicators, Volume 97, 2019.- C. 476-485.

8. Wu Jung-Hua / Electricity portfolio planning model incorporating renewable energy characteristics / Jung-Hua Wu, Yun-Hsun Huang / Applied Energy, Volume 119, 2014.- C. 278-287. 
9. База данных World-Statistics / [Электронный ресурс] URL: http://world-statistics.org/index.php (дата обращения 10.06.2021)

10. Shafieia S. / Non-renewable and renewable energy consumption and CO2 emissions in OECD countries: A comparative analysis / Sahar Shafieia, Ruhul A. Salimb / Energy Policy, Volume 66, 2014 - C. 547-556.

11. Appunn K. / Germany's energy consumption and power mix in charts./ K. Appunn, Y. Haas, J. Wettengel / Clean Energy Wire. Journalism for the energy transition - 21.12.2020 - [Электронный ресурc] URL: https://www. cleanenergywire.org/factsheets/germanys-energy-consumption-and-power-mix-charts (Дата обращения: 01.07.2021)

12. Konnikov, E.A. / Dialectics of the renewable energy market Konnikov, E.A., Konnikova, O.A., Dubolazova, Y.A., Mansurov, R.D. / Proceedings of the European Conference on Innovation and Entrepreneurship, ECIE, 2020, 2020-September, стр. 952-960.

13. Konnikov, E.A. / Oil Industry in Russia: Retrospective Review, Current State and Development Prospects / Konnikov, E., Dubolazova, Y., Zavrichko, O., Malevskaia-Malevich, E. / IOP Conference Series: Materials Science and Engineering, 2020, 940(1), 012027.

14. Бугаева, Т.М. Развитие и реализация возобновляемой энергии в России: проблемы и перспективы / Т. М. Бугаева, А. С. Куропятник, В. В.Бразовская // Неделя науки СПбПУ: Материалы научной конференции с международным участием. Институт промышленного менеджмента, экономики и торговли. В 3-х частях, Санкт-Петербург, 18-23 ноября 2019 года.- Санкт-Петербург: Федеральное государственное автономное образовательное учреждение высшего образования «Санкт-Петербургский политехнический университет Петра Великого», 2019.- С. 154-157.

15. Кичигин, О.Э. Институциональный аспект формирования стратегических ориентиров государственной энергетической политики на региональном уровне при реализации стратегии национальной экономической безопасности / О.Э.Кичигин, Д.Г. Родионов // Экономика и предпринимательство.- 2017. - № 102(87).- С. 394-399.

16. Дмитриев, Н. Д. Оптимизация управленческих процессов в электроэнергетике на основе математического моделирования / Н.Д.Дмитриев, Д.Г. Родионов, С.А. Жильцов // Kant. - 2021. - № 1(38). - C. 18-23. - DOI 10.24923/2222-243Х.2021-38.4.

17. Елистратов В.В. /Выбросы парниковых газов с водохранилищ ГЭС: анализ опыта исследований и организация проведения экспериментов в России / Елистратов В.В., Масликов В.И., Сидоренко Г.И., Молодцов Д.В. / Международный научный журнал Альтернативная энергетика и экология. 2014. № 11 (151). С. 146159.

18. Konnikov, E.A. / The prevalence of renewable energy in the Russian energy market / Konnikov, E.A., Osipova, K.V., Yudina, N.A., Korsak, E.P. / E3S Web of Conferences, 2019, 124, 04018.

19. Правосудов С. / Перспективный газ / Газпром, Корпоративный журнал ПАО «Газпром», № 4, 2020 г. - С. 17 25. 